\title{
Parámetros para el estudio de la producción editorial en contextos de dictadura y emergencia cultural. La editorial Galaxia en el período 1951-1973
}

\section{Parameters for the Study of the Publishing Production in Contexts of Dictatorship and Cultural Emergency. The Galaxia Publishing House in the Period 1951-1973}

\author{
Cristina Martínez Tejero \\ Centro de Estudos Comparatistas. Universidade de Lisboa \\ cristina10@campus.ul.pt \\ ORCID iD: http://orcid.org/0000-0001-8651-4990
}

\section{RESUMEN}

Este artículo tiene como objeto el estudio de la producción de la editorial Galaxia, fundada en Vigo en 1950 y cuya primera obra es publicada en el año siguiente. A través de dos cortes temporales (1951-1967, 1967-1973), se realiza un análisis tipológico y cuantitativo de los títulos editados por esta casa, identificando las tendencias activas y cambios operados durante este período. Se tienen en cuenta tanto el número de ediciones o reediciones, como la lengua, el origen - según los factores formulados por José Lambert: producción, tradición e importación- y la tipología de las obras, realizando en este último caso estudios más detallados sobre las diferentes modalidades de ensayo/libro científico-técnico así como de la creación ficcional. Por último, se ofrecen conclusiones sobre la situación del campo editorial gallego y, en general, del proceso de emergencia del sistema cultural, recalcando la posibilidad de exportación de estos parámetros de análisis a contextos análogos.

Palabras Clave: edición; editorial Galaxia; franquismo; campo literario gallego; emergencia.

\begin{abstract}
The aim of this paper is to study the publishing production of Galaxia, a publishing house founded in Vigo in 1950 and whose first work is published in the following year. Through two temporary sections, 1951-1967 and 1967-1973, a typological and quantitative analysis of the titles published by this house is done, in order to identify the active trends and the changes operated in this period of time. The number of editions or reprints, the language, the origin (according to the factors raised by José Lambert: production, tradition and import) are taken into account. We
\end{abstract}


also address the books' typology, with detailed studies on the different types of essays/scientific-technical works as well as the fictional creation. Finally, we offer conclusions about the situation of Galician publishing field and, in general, about the process of emergence of the cultural system, stressing the possibility of exporting these analysis parameters to similar contexts.

Key words: Publishing; Galaxia Publishing House; Francoism; Galician Literary Field; Emergency.

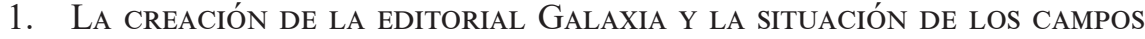 CULTURALES GALLEGOS EN 1950}

La editorial Galaxia fue fundada en Vigo en 1950, catorce años después del golpe franquista que supuso el descalabro de los cuadros galleguistas con el asesinato, encarcelamiento, exilio y acoso de los principales agentes del período de preguerra. Este hecho es paralelo a la destrucción de las principales instituciones culturales creadas hasta ese momento o su continuidad en la etapa franquista desvinculadas de su perfil galleguista, caso el segundo de la Real Academia Gallega o del Seminario de Estudos Galegos, este último transmutado parcialmente en el Instituto Padre Sarmiento, dependiente del gobierno central (Núñez Seixas, 1994; Beramendi y Núñez Seixas, 1996: 187 y ss.). La práctica extinción del Partido Galeguista en la clandestinidad y la ausencia casi total de la lengua $u$ otros símbolos de identidad gallegos en el espacio público (a excepción de un uso folclorizante patrocinado por el proprio franquismo) supuso un cambio en las estrategias utilizadas por el galleguismo a partir de la década de 1950, ante la previsión de la continuidad y estabilidad del régimen de Franco para las próximas décadas (Beramendi y Núñez Seixas, 1996: 191 y ss.).

El conocido como galleguismo del interior (en oposición a aquel que se refugia en América Latina después de la guerra civil y que encuentra en Alfonso Daniel Rodríguez Castelao su gran referente) justifica la creación de la editorial Galaxia ante la imposibilidad de desarrollar una acción política y la necesidad de tener una mínima presencia pública ante la sociedad gallega. La apuesta por un proyecto editorial en estas circunstancias puede ser analizada a partir de dos hechos relacionados: por una parte, en situaciones de dictadura el espacio de posibles (Bourdieu, 1992: 326) está fuertemente condicionado por el campo del poder político, por lo que es habitual que los campos culturales asuman funciones más allá de las propias; por otro, se trata de una propuesta que incorpora el prestigio social asociado al libro en términos notablemente ambiciosos, al ser las casas editoriales una de las iniciativas de mayor envergadura dentro del sector cultural de la época.

Galaxia no es solo una editorial sino también un grupo de actuación político con una influencia notable en el devenir de los campos culturales y la 
configuración política gallega durante la Transición ${ }^{1}$. El motivo de centrar este estudio sobre su producción editorial es ofrecer análisis inéditos sobre el que fue su principal organismo de intervención pública durante sus primeros años y que sirvió de anclaje para la estructuración del grupo.

En estas páginas se ofrecerá un análisis de tipo cuantitativo y cualitativo, con una base metodológica asentada en las teorías sistémicas, fundamentalmente las representadas por la teoría de los campos sociales de Pierre Bourdieu (1992) y del sistema literario de Itamar Even-Zohar (1994 y 2010). En ambos casos se trata de formulaciones con una comprensión extensa del fenómeno literario y de inspiración funcionalista. La situación especial que viven los campos culturales gallegos de la época nos lleva a hablar en algunos puntos de este trabajo de proceso de emergencia, al no haber un sistema estable y consolidado sino hallarse en vías de configuración, con toda la complejidad y ambigüedad que de esto se deriva.

\section{CRiterios y FUENTES PARA El ANÁLISIS DE LA PRODUCCiÓN EDITORIAL DE GalaXIa (1951-1973)}

El marco temporal delimitado para este estudio se corresponde con el período 1951-1973, ocupando ampliamente las dos primeras décadas de la editorial. Dentro de estos límites se procedió a una doble división con base en las características del corpus y en elementos externos relativos a la cronología establecida sobre el régimen franquista que llevan a situar un período específico entre 1968 y 1973, correspondiente al llamado tardofranquismo y sobre el que existen trabajos más amplios (Cordeiro Rua y Samartim, 2008) ${ }^{2}$.

Las fuentes consultadas para fijar las obras publicadas bajo el sello de Galaxia en el período propuesto fueron, en primer lugar y de forma prioritaria, el catálogo de la Biblioteca de la Universidade de Santiago de Compostela (USC), el inventario más completo para esta etapa con relación al libro gallego

${ }^{1}$ Un análisis detallado de la actuación del grupo Galaxia en el período de la Transición puede ser localizado en Samartim (2010). Sobre el surgimiento del proyecto editorial, así como su localización en relación a otras iniciativas editoriales gallegas creadas durante el franquismo, remitimos al estudio de Martínez Martín (2015: especialmente 844 y ss.).

${ }_{2}$ Dadas las modificaciones que tienen lugar en el campo político, al producirse una ligera apertura del régimen y la reactivación de movimientos de oposición, consideramos oportuno defender la conveniencia de utilizar la etiqueta de tardofranquismo en el contexto gallego para el período 1968-1973, correspondiéndose con la etapa iniciada a partir de la emergencia de movilizaciones en la Universidad de Santiago de Compostela y el asesinato del general Carrero Blanco, previsible sucesor de Franco, en diciembre de 1973, dando inicio a la transición, como fase de relocalización en que se detecta un alto grado de tensión y dinamismo en el campo político y que continuaría hasta la aprobación de la Constitución Española en 1978. 
y localizado en una institución que los agentes de Galaxia sitúan como un referente, lo que hace deducir su empeño en tener presencia en su biblioteca ${ }^{3}$. Los posibles errores de catalogación o, incluso, las contradicciones presentadas en algunos de sus datos fueron resueltos mediante el recurso a materiales propios de Galaxia sobre sus publicaciones (Editorial Galaxia, 1970, 1973 y, especialmente, 1974), junto con la eventual consulta física de las obras que así lo requirieron. En lo tocante al período 1968-1973, estas fuentes fueron completadas con la consulta del Catálogo de livros e instituições, Galiza 1968-1978 desarrollada por el proyecto Fisempoga de la USC y disponible para consulta pública en la red, lo que supone la posibilidad de contrastar las cifras de producción de Galaxia y los números totales del campo editorial gallego ${ }^{4}$. La existencia de duplicidades o incoherencias en los catálogos consultados, siendo uno de los problemas más frecuentes la inestabilidad a la hora de atribuir año de publicación a las obras, obligaron asimismo a la revisión y toma de decisiones adaptadas a cada libro en concreto. A pesar de la búsqueda de una coherencia en virtud de la información a las que se tuvo acceso en cada caso, los análisis aquí ofrecidos pretenden más bien reflejar tendencias temporales que defender la precisión absoluta de los datos atribuidos a cada año.

Los aspectos contemplados en este análisis respondieron al siguiente esquema: por una parte, se atendió al número de libros publicados en cada período propuesto y, por otra, se evaluaron las características de las obras desde una perspectiva macro, en tres aspectos específicos, lengua, origen y temática/tipología. En relación al primer ítem mencionado y destinado a medir la capacidad de producción de Galaxia, fueron computadas de forma exhaustiva todas aquellas obras publicadas bajo el sello de la editorial, quedando excluidas tanto las publicaciones periódicas —Revista de Economía de Galicia (1958-1968) y Grial (1963-actualidad)_, así como aquellas obras en que Galaxia actúa simplemente como distribuidora y no como editora ${ }^{5}$. En la medida en que el interés de este cómputo reside sobre todo en la capacidad de publicación de un

${ }^{3}$ La entrada del grupo Galaxia en la Universidade de Santiago de Compostela se produce principalmente a través de la figura de Ricardo Carvalho Calero (en la época, Carballo) desde mediados de la década de 1960 y por medio de un proceso de planificación prolongado visible en diversos epistolarios (Fernández del Riego, 2000; Piñeiro y Losada, 2009).

${ }^{4}$ El equipo del proyecto Fisempoga desarrolló esta base a partir de los datos de la propia USC y la Fundación Penzol, contemplando como obras adscritas al campo editorial gallego aquellas situadas dentro del llamado proyecto sistémico, independientemente de su lengua (Cordeiro Rua y Samartim, 2008: 164 y ss.). Más información en Proyecto Fisempoga (USC). «Catálogo de livros e instituições, Galiza 1968-1978». Disponible en: <http:// www.grupogalabra.com/bases-de-dados/fisempoga.html> [ref. de 4/02/2015].

5 Es el caso de algunas obras atribuidas precisamente a Galaxia en algunos de los catálogos consultados, al tratarse de auto-ediciones, como Estampas gallegas (1961) de José Fernández Méndez o Tres poetas en romería (1973) de Malcom Batchelor, Antonio Carreño y James H. Dwyer. 
mayor número de obras, se contemplan dentro de la cifra aportada las reediciones, que serán objeto de observación específica dentro del período 19681973, dado que se concentran prácticamente en él ${ }^{6}$. En consonancia con lo expuesto, esta operación se mantiene para el cómputo de las características de los libros - es decir, cada obra, sea su primera edición o no, es atendida de forma individual - al entender que el objetivo es ofrecer una lectura proporcional y genérica con relación a otros factores. Un último aspecto a anotar sobre las reediciones es que solo se tienen en consideración aquellos casos en que Galaxia actúe como primera editora y no aquellos en que se trate de una obra proveniente de la tradición, parámetro este ya contemplado en el origen de los libros, según será expuesto a continuación.

En la clasificación de acuerdo con las características de las obras se consideró relevante, en primer lugar, un análisis a partir de la lengua de publicación de los libros, según esta fuese el gallego, el castellano o presentasen un perfil bilingüe, con texto en ambos idiomas ${ }^{7}$. En un segundo nivel, se pretendió evaluar los parámetros dominantes en el origen de las obras editadas por Galaxia a la luz de los tres conceptos introducidos por José Lambert (1980 y 1983) en su análisis de la relación entre sistemas, en que distingue entre producción (actividad literaria en un dado momento y lugar), tradición (actividad literaria del pasado que sobrevive) e importación (procedente de otros sistemas). La aplicación de estos factores en este estudio en concreto tiene como objetivo, por lo tanto, ponderar en qué medida se trata de obras originales, procedentes de etapas previas o son traducciones. Se considera como tradición cualquier libro publicado previamente a la Guerra Civil por entender que funciona como representante del pasado literario, sea este más o menos próximo, así como aquellos asociados a la celebración del Día das Letras Galegas (evento anual destinado precisamente a conmemorar la tradición literaria en lengua gallega me-

${ }^{6}$ La necesidad de actuar de forma coherente con relación a este factor motivó que fuese duplicada en el cómputo correspondiente a 1969 la obra Galicia feudal de Victoria Armesto, que conoció dos ediciones en ese mismo año. Por otra parte, somos conscientes de que el recurso al parámetro de las reediciones puede ser cuestionado a la luz de otros elementos como el número de ventas o tiradas que, sin duda, ofrecerían una lectura mucho más rica del funcionamiento de la editorial en este período. No obstante, la imposibilidad hasta el momento de tener acceso a esa información, junto con la propia relevancia que de por sí tiene el hecho de que una obra conozca más de una edición y las consecuencias que de esto se derivan en términos de articulación del proyecto editorial y de éxito de público nos hace defender, con las precauciones necesarias, los análisis aquí propuestos.

${ }^{7}$ Las obras de perfil artístico en que no existe una extensión de texto lo suficientemente representativa no se contabilizan para la medición de los usos lingüísticos. Por otro lado, en el caso de una obra del profesor portugués Manuel Rodrigues Lapa dedicada a antologizar la poesía medieval, es cuantificada como de lengua gallega, dado que se considera que funciona (en términos sistémicos) dentro del mismo marco lingüístico. 
diante la selección de una figura representativa $)^{8}$. Por otro lado, esta clasificación está relacionada con la procedencia de las publicaciones y no con su temática, por lo que las obras con origen en la literatura popular no computarían como tradición a no ser que hubiesen conocido ediciones previas a Galaxia.

Por último, se desarrolla una ordenación según la tipología temática teniendo en cuenta una primera clasificación inicial de perfil muy amplio y concretada en los siguientes elementos: ensayo o libro científico-técnico, producción literaria, artísticos (con un peso importante del elemento gráfico, incluyendo la reproducción de imágenes), literatura popular y una categoría más extensa para situar aquellas obras que mezclan o rehúyen cualquiera de las clasificaciones anteriores, varios/otros ${ }^{9}$. En lo relativo a la producción ensayístico-científica o literaria, se procede a realizar una clasificación propia dentro de cada una de ellas que atienda a las distintas modalidades o temáticas existentes y concretizada en los siguientes puntos: filosofia, literatura, lengua, historia, economía, etnografíalantropología, pedagogíaleducación u otros para el ensayo y libro científico-técnico; y en poesía, narrativa corta, narrativa larga, teatro, infantil y otros para la producción literaria ${ }^{10}$. En la misma orientación de las elecciones procedimentales ya explicitadas, se optó por la duplicación en aquellos casos en que puede ser verificada más de una tipología, por ejemplo el teatro infantil, que computaría en ambos recuentos.

\section{PERÍODO 1951-1967}

La primera etapa de actividad editorial de Galaxia resulta fundamental para entender su trayectoria, al ser definidas en ella algunas de las líneas directrices que marcarán el rumbo de la editora prácticamente hasta la década de 1980. Durante este período, por ejemplo, se crearon colecciones - entre otras, Illa Nova destinada a autores jóvenes, Trasalba dedicada a clásicos, Salnés de

${ }^{8}$ En este sentido, hay que distinguir entre las obras de autores homenajeados en esta celebración, computables como tradición, de los libros de homenaje realizados ad hoc para esta ocasión, considerados como textos actuales, ejemplo de lo cual es el realizado en 1973 para el arzobispo Lago González y contabilizado como producción. El criterio de la distancia temporal funciona igualmente para clasificar una obra monográfica dedicada al escultor Eiroa (fallecido en 1935) como tradición.

9 Se consideró oportuno concentrar bajo un mismo rótulo tanto los libros ensayísticos como aquellos de perfil más científico o técnico al haber un margen estrecho entre ambos perfiles en la mayoría de las obras contempladas (piénsese, por ejemplo, en la Gramática gallega de Marcial Valladares) y que, en todo caso, operan de forma claramente diferencial en relación con las otras categorías formuladas en esta primera clasificación general.

${ }^{10}$ Dada la existencia de notables diferencias en el número de comparecencias se apreció como necesario la distinción entre narrativa corta (relatos y novelas cortas) y larga (novelas). 
poesía o Manuales - que estructuran la llegada al público de las obras. A nivel operacional, se consideró oportuno efectuar un corte en este período correspondiendo al cambio de década para visualizar mejor las variaciones que se van produciendo en la actividad editorial de Galaxia.

FigurA 1

EDICIÓN DE OBRAS POR AÑO (EDITORIAL GALAXIA, 1951-1967)

Libros editados por Galaxia 1951-1967 | Total: 144

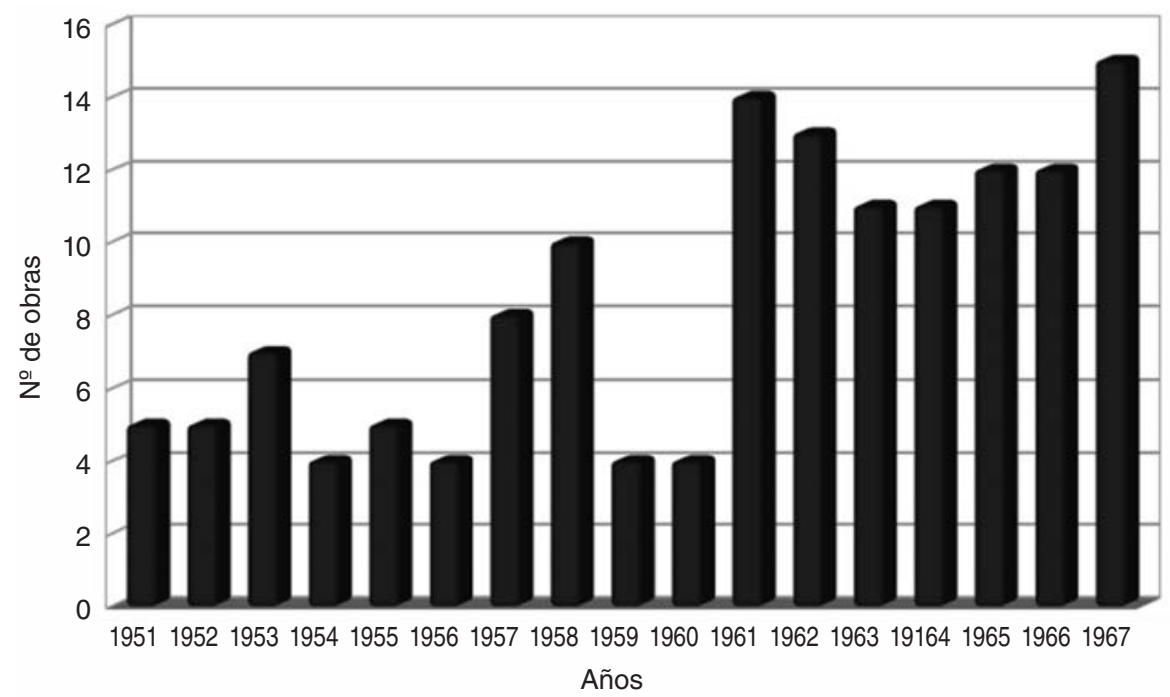

\begin{tabular}{|c|c|c|c|c|c|c|c|c|c|c|c|c|c|c|c|c|c|c|c|}
\hline 1951 & 1952 & 1953 & 1954 & 1955 & 1956 & 1957 & 1958 & 1959 & 1960 & 1961 & 1962 & 1963 & 1964 & 1965 & 1966 & 1967 & Total & $\begin{array}{c}\text { Total } \\
1951-\end{array}$ & $\begin{array}{c}\text { Total } \\
1961- \\
1960\end{array}$ \\
\hline 5 & 5 & 7 & 4 & 5 & 4 & 8 & 10 & 4 & 4 & 14 & 13 & 11 & 11 & 12 & 12 & 15 & 144 & 56 & 88 \\
\hline
\end{tabular}

Elaboración propia.

La producción editorial de Galaxia para el período 1951-1967 [Figura 1] se caracteriza por ser bastante reducida en cuanto al número de volúmenes, con una media total de 8,47 obras por año, pero con un incremento progresivo a medida que se consolida el proyecto y con un notable aumento a partir de la década de 1960, pasando de una media de 5,60 obras por año para el primer corte temporal (1951-1960) a 12,57 en el segundo. Deben ser relativizadas las cifras ofrecidas para los años 1959 y 1960, donde se aprecia un notable des- 
censo del número de ediciones, a la luz de las grandes diferencias constatadas con los años contiguos, 1958 y 1961, dado que se entiende que se produce una transferencia entre ellos. Es decir, libros cuya publicación estaba programada para un año concreto se publican en el año siguiente o también a raíz de las variaciones provocadas por la atribución de fecha de publicación a partir del depósito legal en los catálogos consultados y que resulta en las cifras finalmente obtenidas. Se incluyen en estos cómputos los distintos números correspondientes a la llamada Colección Grial, iniciativa consistente en diversos volúmenes monográficos de autoría múltiple puesta en marcha ante la negativa política para autorizar una publicación periódica con el mismo nombre, la cual vería por fin la luz en 1963 (Fernández del Riego, 1996: 66). En este sentido, se hace oportuno recalcar el esfuerzo editorial que en las condiciones existentes significó la publicación desde 1958 de la Revista de Economía de Galicia y de la propia Grial a partir de los años 60, lo que se concretiza en múltiples problemas e incumplimientos en relación con la periodicidad pretendida en el caso, por lo menos, de la primera (Beiras Torrado, 2005) ${ }^{11}$.

Los usos lingüísticos detectables en la producción editorial de Galaxia en este primer período [Figuras 2 y 3] son representativos de las problemáticas e incertidumbres derivadas de la actitud contraria del gobierno franquista con relación al gallego y la progresiva apertura que tiene lugar en los años 60 (Beramendi y Núñez Seixas, 1996: 195 y s.). Este aspecto puede ser verificado en la voluntad de uso de la lengua gallega, bien sea con productos monolingües o bilingües y en el propio recurso a estos últimos junto con una presencia proporcional mayor del castellano en la primera década, frente a la progresiva desaparición de productos lingüísticos mixtos y una mayor apuesta por el gallego en la segunda.

${ }^{11}$ Existe una obra no computada aquí pero que resulta ejemplificadora tanto de las problemáticas asociadas a la fijación de un corpus de estas características como al propio análisis sobre Galaxia, dada la ambigüedad que domina muchas veces sus actuaciones. Se trata de la traducción de la obra del escritor en español de origen gallego Camilo José Cela, A familia de Pascual Duarte, publicada en 1962 (veinte años después del original) y con intervención de dos galleguistas históricos, Vicente Risco en calidad de traductor y Ramón Otero Pedrayo como prologuista. En el libro físico no existe ninguna mención a Galaxia, sin embargo, en el colofón figuran nombres asociados de forma muy estrecha con el proyecto de la editorial viguesa, «Dirixiron a edición, nas súas tres tiradas, Francisco Fernández del Riego e Ricardo García Suárez» (este último conocido como Xohán Ledo), además de hacer referencia a la impresión en las oficinas gráficas do Faro de Vigo (lugar donde imprime Galaxia). No obstante, en el Almanaque Galaxia 1950-1975 (Editorial Galaxia, 1974) se incluye como una de las publicaciones de la editorial, lo que nos sitúa ante un caso claramente complejo y con varias soluciones posibles: formalmente esta obra no es atribuible a Galaxia pero funcionalmente sí. La opción por no incluirla finalmente en este cómputo responde a la voluntad de trabajar exclusivamente con aquellos productos salidos con el sello editorial de Galaxia y desde la consciencia de que no representa grandes cambios en los análisis propuestos. 
Figuras 2 Y 3

PRODUCCIÓN EDITORIAL DE GALAXIA SEGÚN LENGUA (1951-1967)

Lengua libros 1951-1960

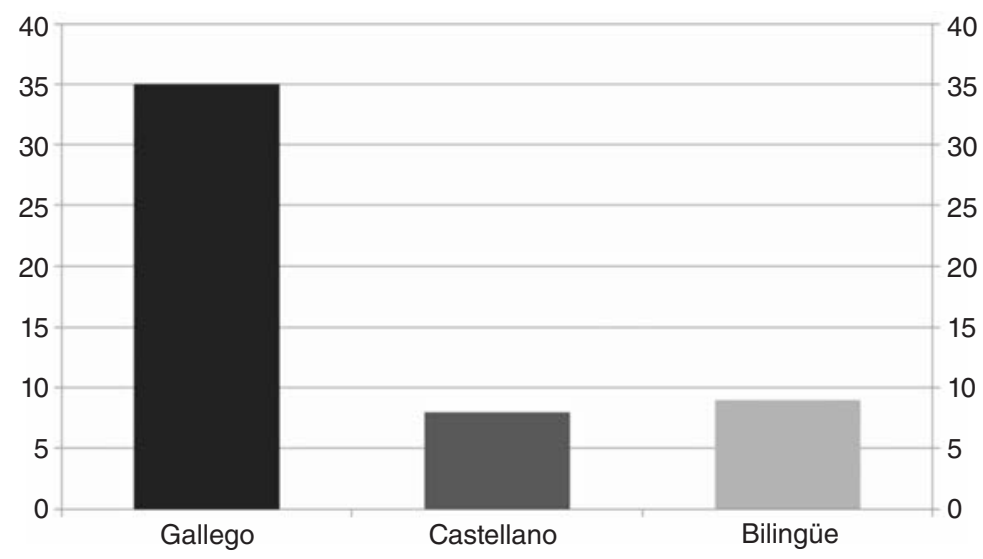

\begin{tabular}{|l|r|}
\hline Gallego & 35 \\
\hline Castellano & 8 \\
\hline Bilingüe & 9 \\
\hline
\end{tabular}

Lengua libros 1961-1967

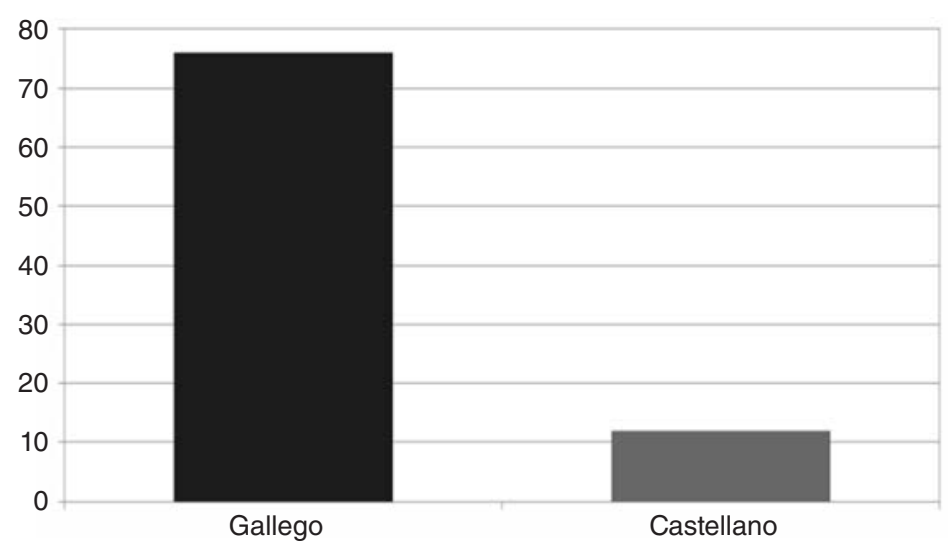

\begin{tabular}{|l|l|}
\hline Gallego & 76 \\
\hline Castellano & 12 \\
\hline
\end{tabular}

Elaboración propia. 
Figuras 4 y 5

PRODUCCIÓN EDITORIAL DE GALAXIA SEGÚN ORIGEN (1951-1967).

Origen libros 1951-1960

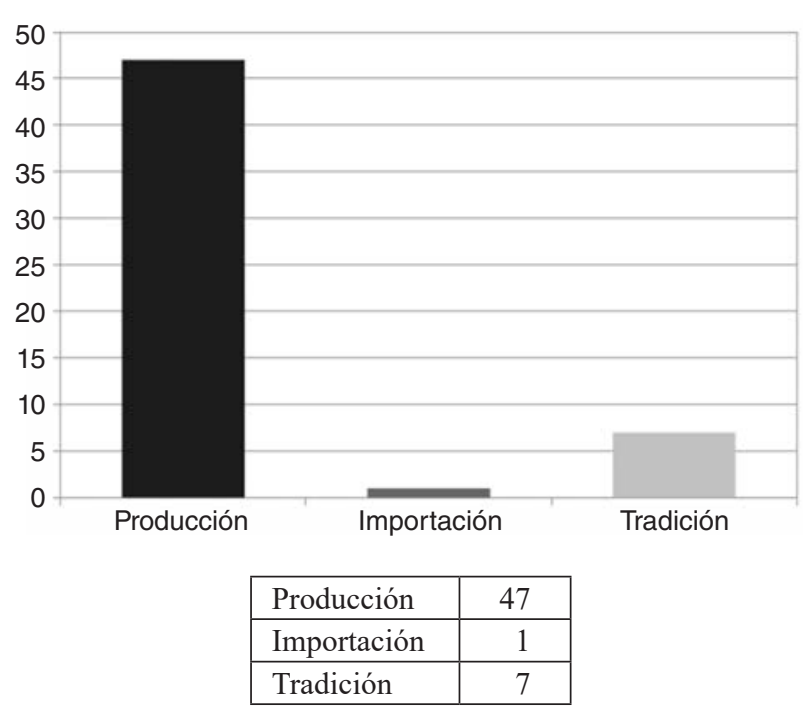

Origen libros 1961-1967

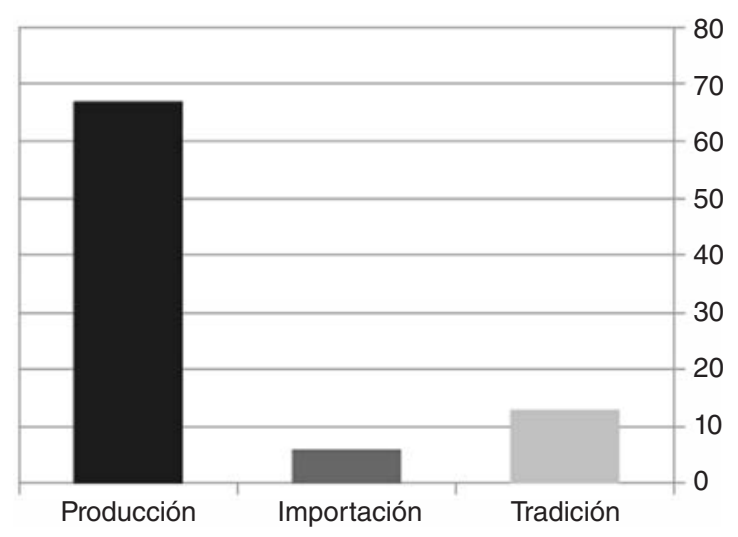

\begin{tabular}{|l|r|}
\hline Producción & 67 \\
\hline Importación & 6 \\
\hline Tradición & 13 \\
\hline
\end{tabular}

Elaboración propia. 
Las obras originales ocupan una posición claramente dominante dentro del catálogo de Galaxia en el período 1951-1967, en claro contraste con los factores de importación y tradición [Figuras 4 y 5]. Esta última se sitúa, no obstante, en un segundo lugar a raíz de la labor de reedición del grupo Nós de la preguerra, preferentemente, y, en un nivel más secundario, del siglo XIX con la recuperación de obras de figuras canónicas del conocido como Rexurdimento de la literatura gallega - Eduardo Pondal y Rosalía de Castro- o, incluso, textos de la época medieval ${ }^{12}$. Sin embargo, la importación experimenta un aumento relativo en la segunda década de la actividad de Galaxia principalmente gracias a la traducción de libros infantiles que complementan la otra vertiente más explotada mediante este mecanismo y relativa a la temática filosófica. Las adscripciones sistémicas originales de las obras traducidas son el sistema alemán en el caso de este último perfil y el catalán - complementado ocasionalmente con el español - en el infantil, producción cuyo despegue en Galaxia tiene lugar en los años finales contemplados en este primer análisis (1966 y 1967) ${ }^{13}$.

El análisis de los datos de producción de Galaxia para el período 1951-1967 [Figuras 6 y 7] evidencia el dominio de la producción literaria y el ensayo (o libro científico-técnico) como modalidades claramente mayoritarias, llegando a abarcar entre ambas más de tres cuartas partes de la producción total. No obstante, y mientras el ensayo o libro técnico se mantienen en la segunda década en cifras próximas a la etapa anterior, pierde peso en comparación con la producción literaria que aumenta de forma notable en el período 1961-1967, pasando de estar prácticamente igualada con el ensayo a constituirse como más de la mitad de la producción de Galaxia. En este sentido, es oportuno recordar que la salida finalmente de la revista Grial implica la desaparición de la colección homónima y la concentración en esta publicación periódica de gran parte de la producción relativa al pensamiento. Por otro lado, tanto la literatura popular como los libros artísticos continúan manteniendo números semejantes, si bien contribuye a ello, en el último caso citado, la edición de diversas obras de ilustraciones de la autoría de Castelao salidas entre 1961 y 1967 (como varios números de la serie Cousas da vida), mientras que se reducen las dominantes en la década anterior, dedicadas a la reproducción gráfica de obras pictóricas o escultóricas de artistas gallegos.

En lo que concierne a las obras de orientación más analítica y técnica [Figuras 8 y 9], hay que anotar la reducción significativa de las temáticas filosó-

12 Bajo el nombre de grupo Nós se hace referencia al colectivo literario fraguado en torno a la revista del mismo nombre publicada entre 1920 y 1936. Por otra parte, la historiografía literaria gallega consagró la etiqueta de Rexurdimento para la segunda mitad del siglo XIX, en que se considera que hubo una reactivación de la escrita en gallego después de tres siglos de ausencia, los llamados «Séculos escuros».

13 Un estudio sobre la literatura infantil gallega en el tardofranquismo puede ser localizado en Figueiras (2009). 
Figuras 6 y 7

PRODUCCIÓN EDITORIAL DE GALAXIA SEGÚN TIPOLOGÍA (1951-1967).

Tipología libros 1951-1960 (I)

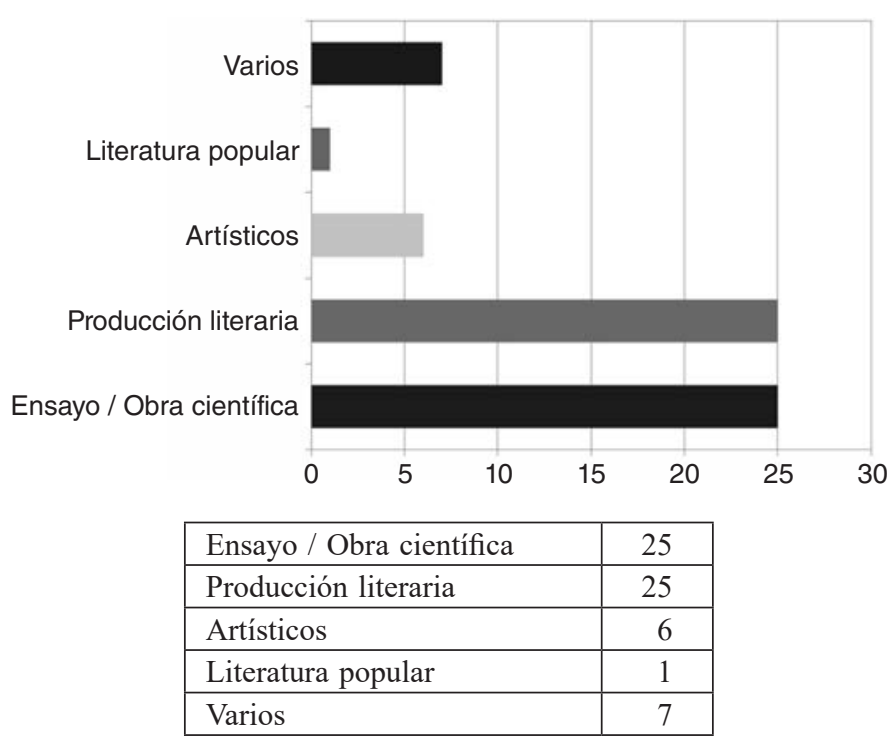

Tipología libros 1961-1967 (I)

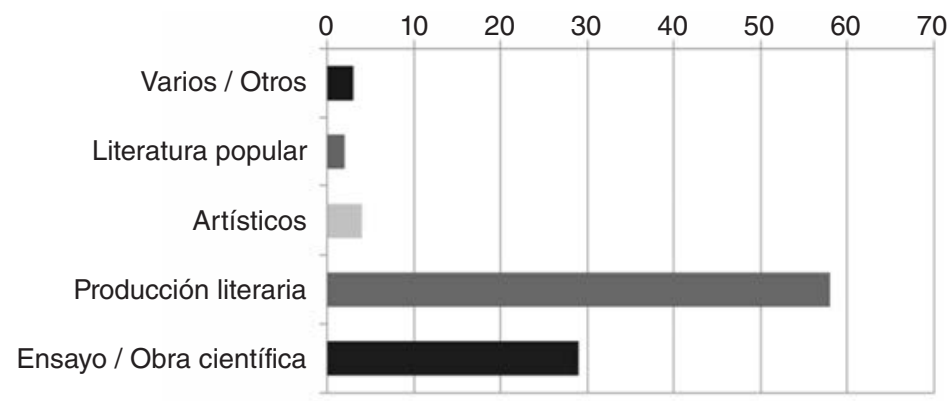

\begin{tabular}{|l|c|}
\hline Ensayo / Obra científica & 29 \\
\hline Producción literaria & 58 \\
\hline Artísticos & 4 \\
\hline Literatura popular & 2 \\
\hline Varios / Otros & 3 \\
\hline
\end{tabular}

Elaboración propia. 
Figuras 8 y 9

EL ENSAYO Y LIBRO CIENTÍFICO-TÉCNICO EN LA PRODUCCIÓN EDITORIAL DE GALAXIA (1951-1967)

Tipología libros 1951-1960 (II). Ensayo y libro cientifico

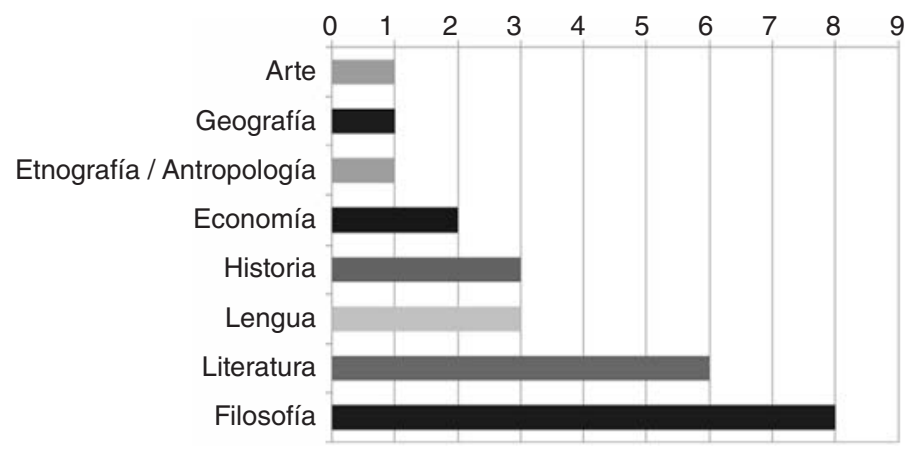

\begin{tabular}{|l|l|l|l|}
\hline Filosofía & 8 & Economía & 2 \\
\hline Literatura & 6 & Etnografía / Antropología & 1 \\
\hline Lengua & 3 & Geografía & 1 \\
\hline Historia & 3 & Arte & 1 \\
\hline
\end{tabular}

Tipología libros 1961-1967 (II). Ensayo y libro cientifico

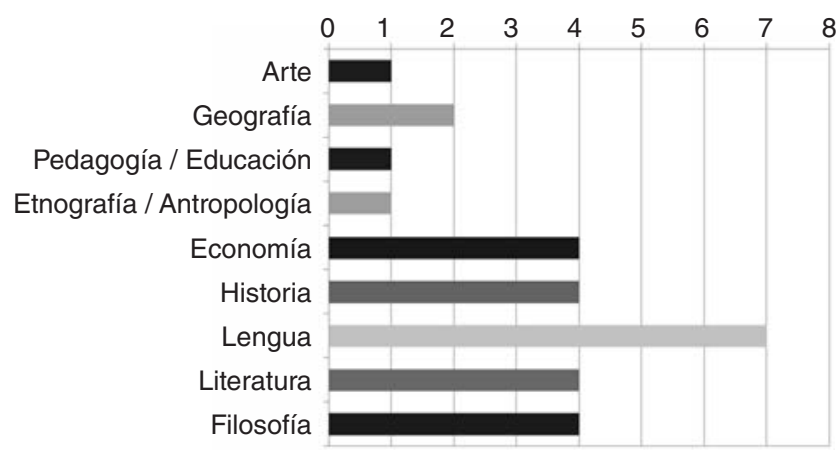

\begin{tabular}{|l|l|l|l|}
\hline Filosofía & 4 & Etnografía / Antropología & 1 \\
\hline Literatura & 4 & Pedagogía/Educación & 1 \\
\hline Lengua & 7 & Geografía & 2 \\
\hline Historia & 4 & Arte & 1 \\
\hline Economía & 4 & & \\
\hline
\end{tabular}

Elaboración propia. 
fica y literaria que se produce en la segunda década estudiada, posiblemente porque la revista Grial concentra esos mismos intereses, lo que se traduce, como fue indicado, en la publicación de artículos en esta revista y la reducción del número de libros. Del mismo modo, el dominio de estos asuntos en el períodos 1951-1960 — en que ocupan más de la mitad de la producción dentro de este perfil - es claramente representativa de la preferencia dada desde el grupo Galaxia a estas formulaciones, con un privilegio evidente de cuestiones relativas a la alta cultura, concretizadas en el tratamiento de asuntos filosóficos y literarios. En el período 1961-1967 tiene lugar un incremento más que notable de la producción relativa a la lengua lo que va nuevamente en consonancia con las propuestas grupales en torno a este elemento, considerado central en la definición identitaria gallega y en relación con la cual hay un interés manifiesto por darle soporte científico ${ }^{14}$. También en aumento, pero sin una proporción tan significativa, figura la temática económica, lo que debe ser relacionado con la continuidad de los esfuerzos desarrollados desde la publicación periódica monográfica sobre este asunto ya referida. Finalmente, las obras de asunto histórico se mantienen en unas cifras semejantes e importantes en lo global; sin embargo, es oportuno anotar el escaso cultivo de la rama antropológico-etnográfica, lo cual puede ser explicado por el privilegio concedido a esta temática desde orientaciones subsistémicas, tendentes a la inserción del sistemas literario gallego dentro del sistema español, en oposición a las inclinaciones protosistémicas del grupo Galaxia, defensor de la consolidación de un sistema autónomo ${ }^{15}$.

En lo que concierne a la producción literaria [Figuras 10 y 11], una observación de todo el período permite confirmar la importancia del género poético, incluso con un aumento notable a partir de 1961 en que alcanza más de la mitad de la producción literaria de Galaxia. La narrativa se sitúa en un segundo lugar con un dominio de sus modalidades de extensión corta, frente a la situación deficitaria observable en la primera década en relación con la novela. Esta experimenta un aumento significativo en los años 60 como consecuencia de la importación de repertorios vigentes en sistemas consolidados, principalmente el francés, efectuada sobre todo por agentes jóvenes (que posteriormente serán encuadrados dentro del movimiento de renovación conocido a nivel crítico como «Nova narrativa»). En este sentido, es posible detectar la tendencia a que las nuevas productoras y productores que se incorporan al campo literario opten por los géneros de la poesía y el relato como modalidades preferentes para sus primeras obras, sin que estas sean, lógicamente, exclusivas de una promoción joven de agentes, sino que su cultivo se extiende a figuras de

14 Para un tratamiento de las propuestas de Galaxia en torno a la lengua gallega puede ser consultado Samartim (2005).

15 Las nociones de tendencias proto y subsistémicas fueron desarrollas por Torres Feijó (2004), quien ofrece numerosas claves para su comprensión. 
FIGURAS 10 Y 11

LA CREACIÓN FICCIONAL EN LA PRODUCCIÓN EDITORIAL

DE GALAXIA (1951-1967)

Tipología de libros 1951-1960 (III). Producción literaria

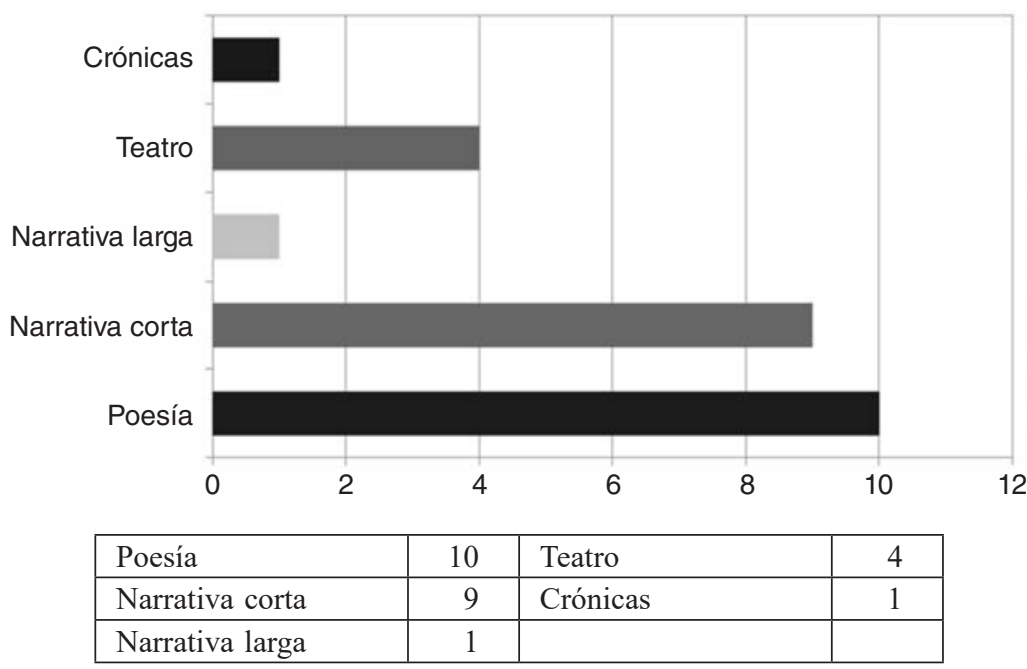

Tipología libros 1961-1967 (III). Producción literaria

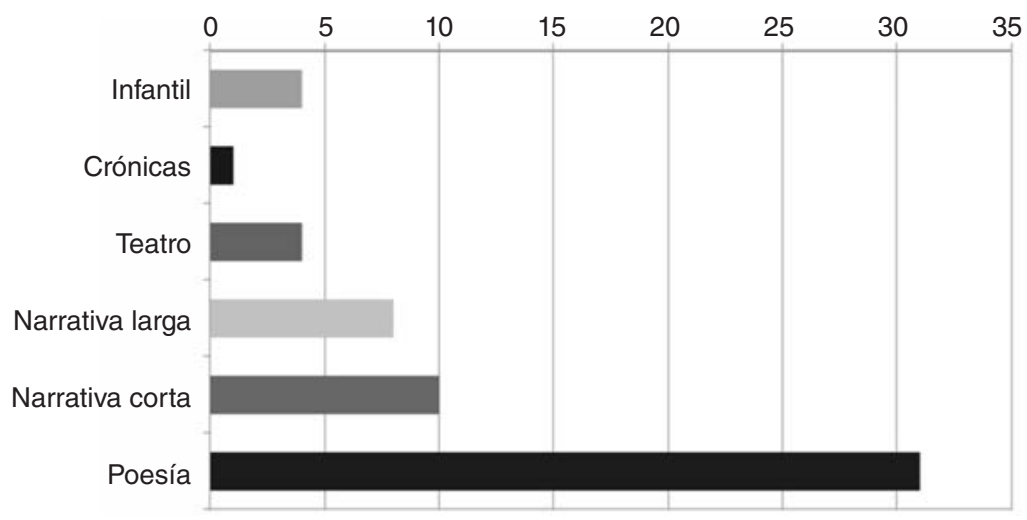

\begin{tabular}{|l|r|l|l|}
\hline Poesía & 31 & Teatro & 4 \\
\hline Narrativa corta & 10 & Crónicas & 1 \\
\hline Narrativa larga & 8 & Infantil & 4 \\
\hline
\end{tabular}

Elaboración propia. 
más edad o con una mayor consolidación. En lo relativo al teatro, este se mantiene en unas cifras análogas en las dos etapas definidas y con una posición muy desigual y deficitaria con relación a los otros géneros atendidos, mientras se observa la aparición de productos destinados al público infantil a partir del segundo lustro de la década de 1960.

\section{Período 1968-1973}

Desde la posibilidad de efectuar una lectura contrastada entre la producción de Galaxia y el total de obras editadas en el sistema durante el período comprendido entre 1968 y 1973, se ofrece a continuación un análisis más detallado de esta etapa. Tal como ha sido indicado, los datos sobre el campo editorial gallego tienen como origen la base de datos sobre libros habilitada por el proyecto Fisempoga de la USC para el contexto gallego durante el período 1968-1978.

En relación con las cifras totales del campo editorial gallego se detecta un incremento progresivo en este período, con un crecimiento proporcional menor entre los años 1970 y 1971, posiblemente a raíz de la crisis del petróleo a nivel mundial que tiente importantes consecuencias, directas o indirectas, en todos los sectores económicos. Esta tendencia no tiene equivalencia con los números de Galaxia que presentan subidas y bajadas más o menos constantes y que nos sitúan ante una media de 22,8 libros por año, cantidad muy superior a la observada en períodos previos [Figuras 12 y 13]. Cabe destacar, asimismo, la colaboración de la editorial viguesa con otras instituciones culturales y de la cual resultan publicaciones que engrosan el catálogo de Galaxia, como las seis obras subsidiadas por la Fundación Penzol ${ }^{16}$ entre 1968 y 1973 —salidas en algún caso de los seminarios científicos por ella promovidos y dominando entre ellas el perfil filológico_-, o el libro Lecturas gallegas 1 (1972), integrante del proyecto pedagógico desarrollado por el Instituto da Lingua Galega, organismo creado en la USC para la elaboración de materiales para la enseñanza. A esto deben ser sumadas la edición anual de obras relativas o de la autoría de la persona homenajeada en el Día das Letras Galegas, evento dependiente de la Real Academia Gallega pero impulsado en su origen por agentes de Galaxia activos en esta institución.

16 La Fundación Penzol fue creada en Vigo en 1963 con la intención de promover las investigaciones sobre la realidad gallega y teniendo como base la biblioteca del galleguista histórico Fermín Penzol Labandeira. El surgimiento y evolución de esta iniciativa guarda una estrecha relación con Galaxia, cuyos miembros ejercieron como promotores y ocuparon cargos relevantes en sus órganos directivos. A pesar de esta relativa sincronía de actuación, consideramos oportuno marcar las obras del catálogo de Galaxia promovidas por esta institución por ser ejemplos tanto de la colaboración entre ambos organismos como de las líneas de actuación privilegiadas desde la Fundación Penzol. 
Figuras 12 y 13

PRODUCCIÓN EDITORIAL DE GALAXIA EN CONTRASTE

CON EL CAMPO EDITORIAL GALLEGO (1968-1973)

Producción editorial de Galaxia (1968-1973)

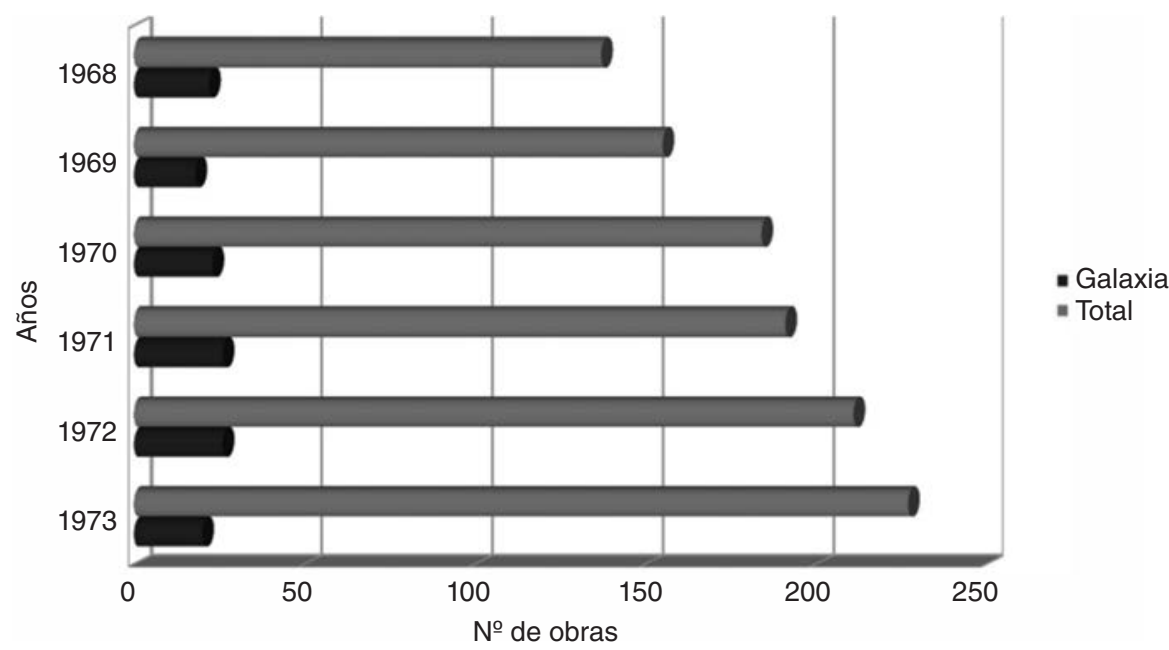

Impacto de Galaxia en el campo editorial gallego según ediciones (1968-1973)

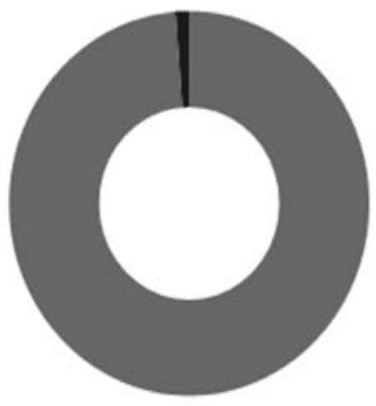

- Galaxia

- Total

\begin{tabular}{|l|c|c|c|c|r|r|r|}
\hline & $\mathbf{1 9 6 8}$ & $\mathbf{1 9 6 9}$ & $\mathbf{1 9 7 0}$ & $\mathbf{1 9 7 1}$ & $\mathbf{1 9 7 2}$ & $\mathbf{1 9 7 3}$ & \multicolumn{1}{|c|}{ Total } \\
\hline Galaxia & 22 & 18 & 23 & 26 & 26 & 20 & 137 \\
\hline Total & 137 & 155 & 184 & 191 & 211 & 227 & 11.060 \\
\hline
\end{tabular}

Elaboración propia. 
El contraste ente el número total de obras editadas por Galaxia y la cifra correspondiente a la suma de la producción en el campo editorial gallego para estos años confirma el impacto pequeño de la editorial de Vigo dentro de estos cálculos aunque, para ofrecer una evaluación rigorosa, sería necesario contrastar estos datos con informaciones adicionales que den cuenta, por ejemplo, de la configuración del resto del panorama editorial asociado a Galicia (tanto si se trata de empresas afincadas en este espacio o españolas con publicaciones sobre tema gallego), junto con cifras sobre tiradas o ventas e, incluso, cuestiones de diseño, distribución o acción de la censura, entre otras ${ }^{17}$.

Figura 14

REEDICIONES EN LA PRODUCCIÓN EDITORIAL DE GALAXIA

(1951-1973)

Número de reediciones de Galaxia (1951-1973)

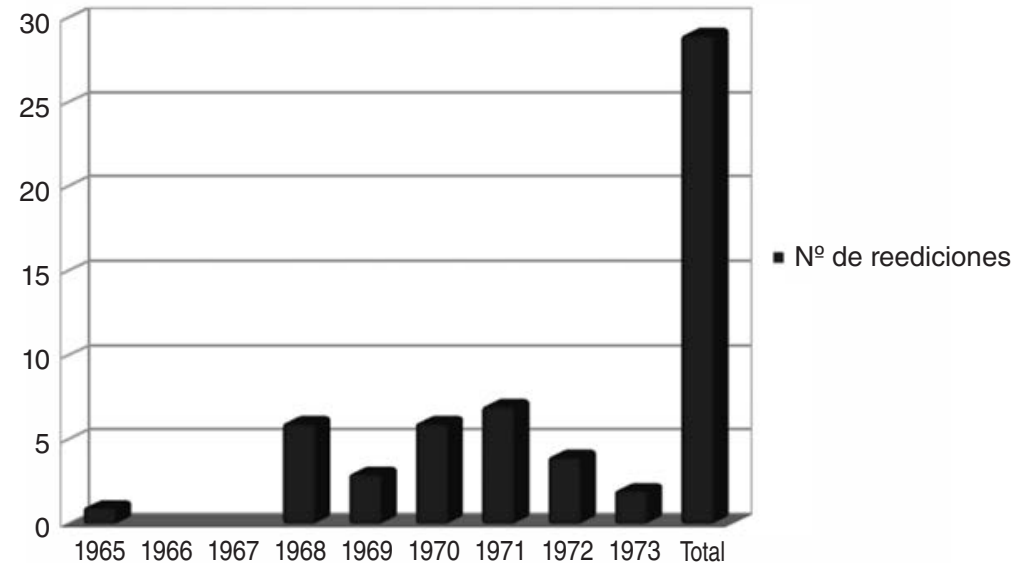

\begin{tabular}{|l|c|c|c|c|c|c|c|c|c|c|}
\hline Años & $\mathbf{1 9 6 5}$ & $\mathbf{1 9 6 6}$ & $\mathbf{1 9 6 7}$ & $\mathbf{1 9 6 8}$ & $\mathbf{1 9 6 9}$ & $\mathbf{1 9 7 0}$ & $\mathbf{1 9 7 1}$ & $\mathbf{1 9 7 2}$ & $\mathbf{1 9 7 3}$ & Total \\
\hline N. $^{\mathbf{0}}$ de reediciones & 1 & 0 & 0 & 6 & 3 & 6 & 7 & 4 & 2 & 29 \\
\hline
\end{tabular}

Elaboración propia.

17 Por dar alguna información en este sentido, el estudio desarrollado por Cordeiro Rua y Samartim (2008) para el período 1968-1978 apunta un peso mayoritario de las pequeñas editoriales en la edición de libro en gallego, mientras que la producción de libro (gallego) en español estaría dominada por la edición institucional. 
El aumento de la producción de Galaxia durante el período 1968-1973 debe ser puesto en relación con el recurso a la reedición de obras que habían visto la luz en este sello [Figura 14]. La primera reedición tiene lugar en 1965, quince años después de la fundación de la editorial, y es una práctica que se generaliza a partir de 1968. Se trata de publicaciones con un buen funcionamiento en cuanto a ventas y cuyos rendimientos económicos son importantes para la manutención de la editorial. Entre la nómina de libros sometidos a este proceso destacan por su presencia reiterada las obras de autoría de Afonso Daniel Rodríguez Castelao, que suman prácticamente un tercio del número total de reediciones e, incluso, con casos tan significativos como la obra de teatro Os vellos non deben de namorarse que presenta en el período seleccionado hasta tres ediciones distintas (correspondientes a 1952, 1968 y 1973) ${ }^{18}$. Otras obras de la tradición reeditadas en este momento se corresponden con la Guía de Galicia de Otero Pedrayo (primera edición de 1926 y, en Galaxia, 1954 y 1965), Dos arquivos do trasno de Rafael Dieste (primera edición de 1926 y, en Galaxia, 1962 y 1973), la obra canónica del Rexurdimento Cantares gallegos de Rosalía de Castro (1863 y con ediciones en Galaxia en 1963 y 1970) o la edición revisada y ampliada en relación con la publicada en 1965 de la antología de Rodrigues Lapa Cantigas d'escarnho e de mal dizer dos cancioneiros medievais galego-portugueses (1970). Igualmente obras originales asociadas a nombres de la etapa de preguerra tienen éxito en este momento, como demuestran las segundas ediciones de Prosas galegas de López Cuevillas (1962 y 1971) o Leria (1961 y 1970) y el Manual de historia de Galicia (1952 y 1971), ambas de Vicente Risco.

Entre los libros de nueva producción cabe destacar la figura de Álvaro Cunqueiro del cual se reeditan dos obras - Merlín e familia i outras historias (1955 y 1968) y Escola de menciñeiros e fábula de varia xente (1960 y 1969)-, al que se unen otros nombres y publicaciones que alcanzan un impacto notable en este período como A lús do candil de Ánxel Fole (1953 y 1968), Villardevós de Silvio Santiago (1961 y 1970), Os Biosbardos de Blanco-Amor (1962 y 1970), Xente no rodicio de Neira Vilas (1965 y 1971) o, en el ámbito no ficcional, Galicia feudal de Victoria Armesto con dos ediciones en 1969. Entre las temáticas que concitan un mayor interés para el público lector están las relativas a la literatura popular — con reediciones del Cancioneiro popular gallego de Ramón Cabanillas (1951 y 1972) ${ }^{19}$ y Contos populares de la provincia de Lugo (1963 y 1972)—, y la lengua, con un dato significativo que son

${ }^{18}$ En los años se 60 se produce en Galicia una popularización de Castelao, líder intelectual del galleguismo de preguerra y fallecido en el exilio en 1950, sobre todo a raíz de representaciones de la obra teatral citada (véase Torres Regueiro, 2010 o Dasilva, 2007).

${ }^{19}$ La versión de 1951 — primer libro publicado por Galaxia- lleva por título Antífona de la cantiga, lo que no resulta transparente del contenido de la obra consistente en una antología de poesía popular. 
las 3 ediciones (la cuarta es de 1974) de la Gramática elemental del gallego común de la autoría de Ricardo Carvallo (en la época, Carballo) Calero (1966, 1968 y 1970) o la reedición del Diccionario gallego-castelán y vocabulario castelán-gallego de Franco Grande (1968 y 1972), cuya última parte conoce, además, una publicación independiente como Vocabulario gallego-castelán (1972), y que se suman, en su calidad de libros especializados o de consulta, a la colección de manuales, de la cual ya fue mencionado el de Risco relativo a la historia pero al que debe ser añadido el Manual de Historia de la Literatura gallega (1971) de Fernández del Riego, publicado en esta ocasión en gallego y con ampliaciones en relación con su versión de 1951.

FiguRA 15

PRODUCCIÓN EDITORIAL DE GALAXIA POR LENGUA (1968-1973)

Producción de Galaxia según lengua (1968-1973)

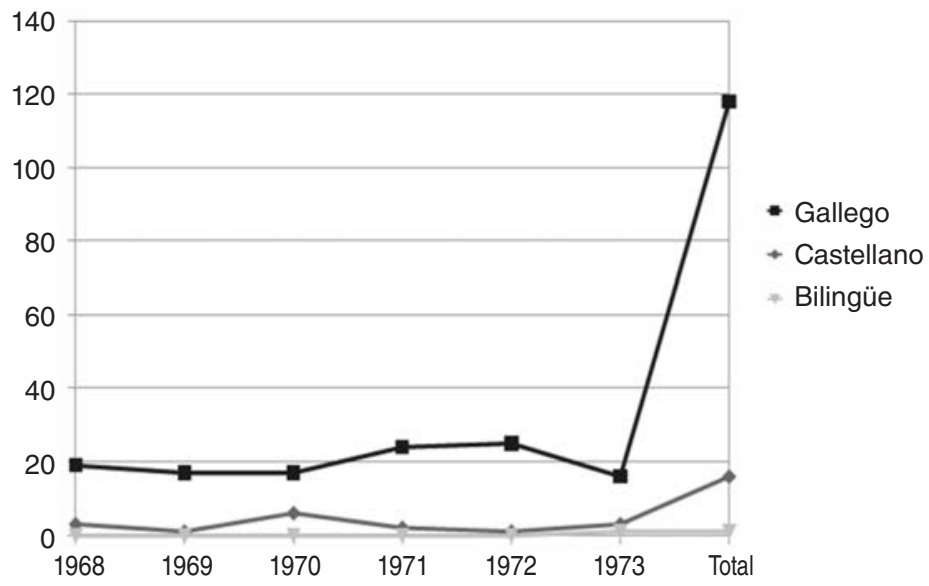

\begin{tabular}{|l|c|c|c|c|c|c|c|}
\hline & $\mathbf{1 9 6 8}$ & $\mathbf{1 9 6 9}$ & $\mathbf{1 9 7 0}$ & $\mathbf{1 9 7 1}$ & $\mathbf{1 9 7 2}$ & $\mathbf{1 9 7 3}$ & Total \\
\hline gallego & 19 & 17 & 17 & 24 & 25 & 16 & 118 \\
\hline castellano & 3 & 1 & 6 & 2 & 1 & 3 & 16 \\
\hline bilingüe & 0 & 0 & 0 & 0 & 0 & 1 & 1 \\
\hline
\end{tabular}

Elaboración propia.

El análisis de la producción editorial de Galaxia en el período propuesto [Figura 15] demuestra la tendencia ampliamente mayoritaria para la publicación de obras en gallego, cuya proporción con relación al castellano es mucho mayor que en períodos previos, si bien continúan existiendo obras no ficcionales publi- 
FIGURA 16

PRODUCCIÓN EDITORIAL DE GALAXIA POR ORIGEN (1968-1973)

Origen obras (1968-1973)

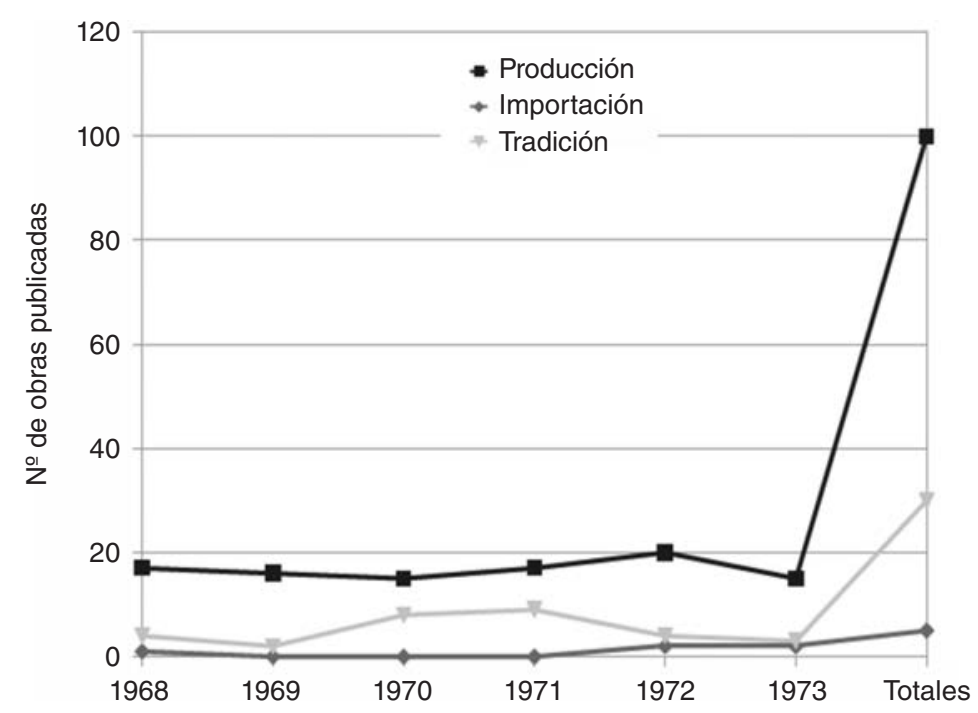

\begin{tabular}{|l|c|c|c|c|c|c|c|}
\hline & $\mathbf{1 9 6 8}$ & $\mathbf{1 9 6 9}$ & $\mathbf{1 9 7 0}$ & $\mathbf{1 9 7 1}$ & $\mathbf{1 9 7 2}$ & $\mathbf{1 9 7 3}$ & Totales \\
\hline Producción & 17 & 16 & 15 & 17 & 20 & 15 & 100 \\
\hline Importación & 1 & 0 & 0 & 0 & 2 & 2 & 5 \\
\hline Tradición & 4 & 2 & 8 & 9 & 4 & 3 & 30 \\
\hline
\end{tabular}

Elaboración propia.

cadas en esa lengua, con un cierto impacto en el caso de aquellas subsidiadas por la Fundación Penzol y que, en ocasiones, recogen trabajos de estudiosos extranjeros, tal como ejemplifica la obra La Pastorela medieval hispánica. Pastorelas y serranas galaico-portuguesas de Arlene T. Leser (1970). Cabe destacar, asimismo, la práctica extinción del perfil bilingüe entre estas publicaciones, si bien hay que matizar esta afirmación con referencia al poemario Río Tambre. Libro das túas soidades ou cantos e prantos de amor de Xosé María García Rodríguez (1973), en que figuran las versiones en gallego y castellano de los mismos poemas, lo que es significativo dada la tendencia al monolingüismo en gallego de Galaxia en la producción literaria, incluso en etapas anteriores ${ }^{20}$.

20 El propio autor, García Rodríguez (1973: 9), justifica este hecho por su trayectoria vital en el extranjero: «Cheo de ledicia o meu ventureiro curazón, poño nas vosas mans iste 
Los parámetros identificados para períodos previos continúan siendo válidos en este caso en que las obras de (nueva) producción prolongan su dominio con una amplia mayoría [Figura 16]. La explotación de la tradición proviene tanto de las publicaciones asociadas a la celebración del Día das Letras Galegas (si bien en algunos casos en que se formulan como libros de homenaje fueron valorados como producción en este estudio), como sobre todo por la recuperación de textos considerados relevantes en la historia de la literatura gallega o que eventualmente puedan tener un buen funcionamiento a nivel de ventas. Como es deducible de lo anotado para las reediciones, las obras de Castelao ocupan un protagonismo importante y en algunos casos justifican las cifras verificadas para la tradición (por ejemplo, en 1971 se editaron cuatro volúmenes pertenecientes a la serie «Cousas da vida»). En un segundo nivel e incorporando este nombre, debe ser anotado el peso general de la producción literaria asociada a la etapa de preguerra y de forma más secundaria al Rexurdimento, mientras que la etapa medieval u otros períodos presentan una comparecencia menos relevante. Hay que añadir el recurso especialmente a obras de perfil literario y entre las que destaca, más allá de la presencia del género poético -dominante en muchas de las etapas contempladas-, un interés notable por productos narrativos, sobre todo los de extensión media (relato largo) y amplia (novela), posiblemente para superar las propias carencias detectables con relación a la producción de obras de este perfil durante el período abordado.

Por otro lado, la importación está principalmente asociada a dos factores consistentes, por una parte, en el aumento, ya detectado en los años previos, de la literatura destinada al público infantil; esto se plasma en la traducción de obras ya no procedentes del sistema catalán (substituidas ahora por producción propia), sino algunos libros canónicos de este perfil como es el caso de $O$ principiño de Saint-Exupéry — con versión gallega (1972) de uno de los autores emergentes y que acabaría por tener un papel protagonista en Galaxia en los años futuros, Carlos Casares-. Un segundo elemento con peso en la selección de obras a traducir son las propias deficiencias apreciadas en relación con la producción en el género dramático y que se concretiza en la elección de obras de autores prestigiados a nivel internacional, como son $A$ traxedia de Macbeth de William Shakespeare y Morte na catedral de T. S. Eliot, o, incluso, la combinación con el factor anteriormente referido, con la elección de obras teatrales destinadas la público infantil como Os anxos cómense crús del chileno Jorge Díaz. Un caso aparte dentro de la importación estaría representado por las Cartas de Galicia de la autoría del periodista belga Prosper-Henri Devos (1889-1914), que escribió estas crónicas en su calidad de corresponsal a inicios del siglo XX para el periódico L’Étoile Belge y en las que aborda la

libro. Inda que esquirto por galego e pra galegos, oferece as miñas traduciós. Ao ter cáseque trinta anos fora de España, son moitos os meus amigos e amigas que non o entenderían na língua da terra». 
Figuras 17 y 18

PRODUCCIÓN EDITORIAL DE GALAXIA POR TIPOLOGÍA (1968-1973)

Tipología libros 1968-1973 (I). Clasificación general

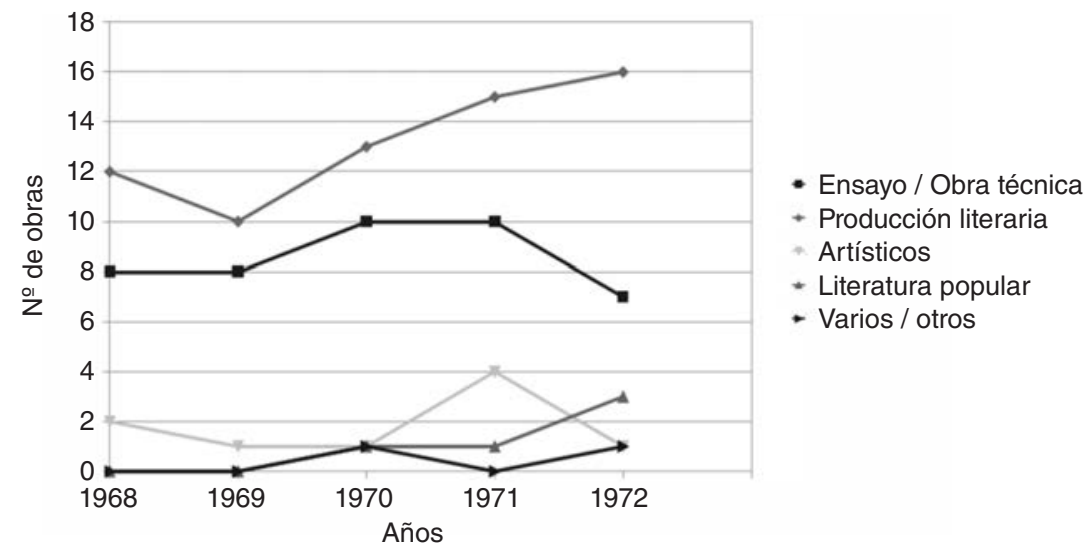

Tipología libros 1968-1973 (I)

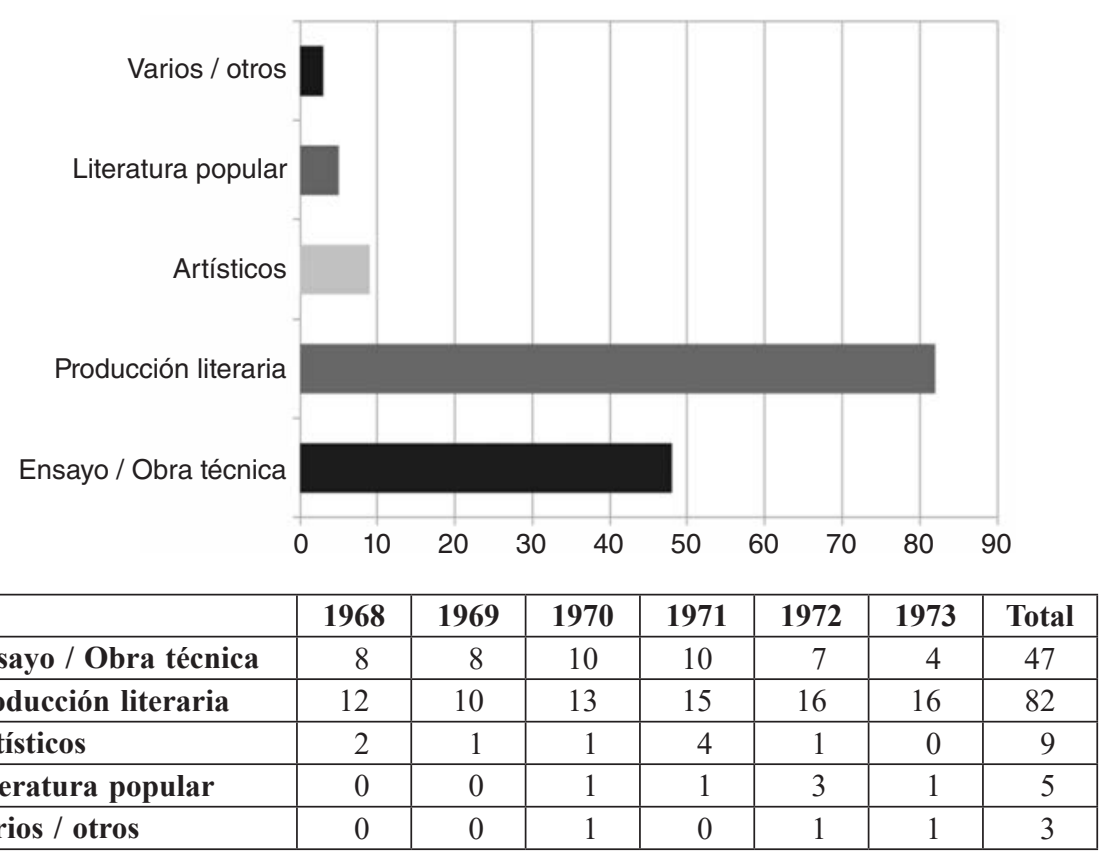

Elaboración propia. 
realidad de la Galicia de la época. La versión gallega — habían sido publicadas en 1913 en castellano en el diario La centuria de Ourense-, que ve la luz en Galaxia en 1968, es de la responsabilidad del intelectual galleguista Xosé Ramón Fernández Oxea, más conocido como Ben-Cho-Shey. Es posible atribuir un doble valor a esta obra, el documental y el legitimador, al ser ejemplificadora de la atracción de intelectuales europeos por la realidad gallega, muy en la línea de la actuación del grupo Nós de la preguerra, siempre interesados en poner de relieve las conexiones de Galicia con Europa.

Al igual que en períodos previos, la producción literaria se perpetua como la modalidad claramente mayoritaria en las cifras de edición de Galaxia entre 1968 y 1973, seguida, aunque con bastante diferencia, por la producción ensayística o científico-técnica [Figuras 17 y 18]. Las otras categorías —más específicas y menos abarcadoras en su definición- presentan un grado de comparecencia mucho menor en comparación con las mencionadas pero manteniéndose dentro de unas cifras no muy discordantes con las apreciadas para etapas previas, si bien con un ligero aumento de la producción clasificable como «literatura popular» y una manutención de la «artística», gracias fundamentalmente a las obras de Castelao de orientación gráfica.

El contraste entre los datos relativos al ensayo y libro científico-técnico del período abordado y los previos ofrece algunas informaciones relevantes [Figuras 19 y 20]. En primer lugar, se constata la decadencia de dos señas de identidad temática del grupo Galaxia como son la filosofía (de forma muy significativa dado que la cifra computada corresponde a dos volúmenes de una misma obra, Dialéctica existencial y psicoanálisis de López Nogueira) y la literatura (con un descenso proporcional no tan abultado). Esta es una tendencia ya verificada en la etapa entre 1961 y 1967, con relación al gran impacto de estas áreas en la primera década de actividad de Galaxia, y que se justifica, en parte y como fue indicado, debido a que mucha de la producción de este perfil se publicó en forma de artículos en la revista Grial. Otra hipótesis para justificar este cambio tiene que ver con la progresiva desaparición como autores de los integrantes principales del grupo (especialmente Ramón Piñeiro) a medida que se consolida una nueva generación de productores y productoras en el sistema y no existe una necesidad tan evidente de publicar obras propias.

Por otro lado, la lengua y la historia se configuran como las temáticas con mayor éxito, seguidas de cerca por la economía que experimenta un aumento de obras monográficas, posiblemente a raíz de la desaparición de la Revista de Economía, cuyo último número corresponde al año 1968. Mientras la lengua se mantiene en unos niveles análogos, existe un incremento notable de la historia en relación con etapas previas (lo que contrasta nuevamente con la posición minoritaria de las obras de orientación etnográfica). Finalmente, debe ser confirmada una tendencia ya apreciada a finales de la etapa de 1961-1967 consistente en el avance de los libros de perfil pedagógico, especialmente combinados con la didáctica de la lengua, ante la previsión - y posterior confirma- 
Figuras 19 y 20

EL ENSAYO Y LIBRO CIENTÍFICO-TÉCNICO EN LA PRODUCCIÓN DE GALAXIA (1968-1973)

Tipología libros 1968-1973 (II). Ensayo y libro cientifico-técnico

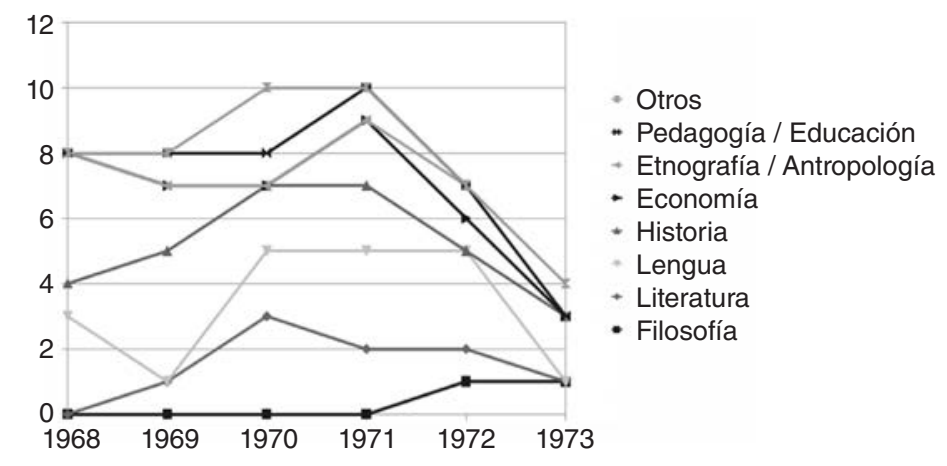

Tipología libros 1968-1973 (II). Ensayo y libro cientifico-técnico (totales)

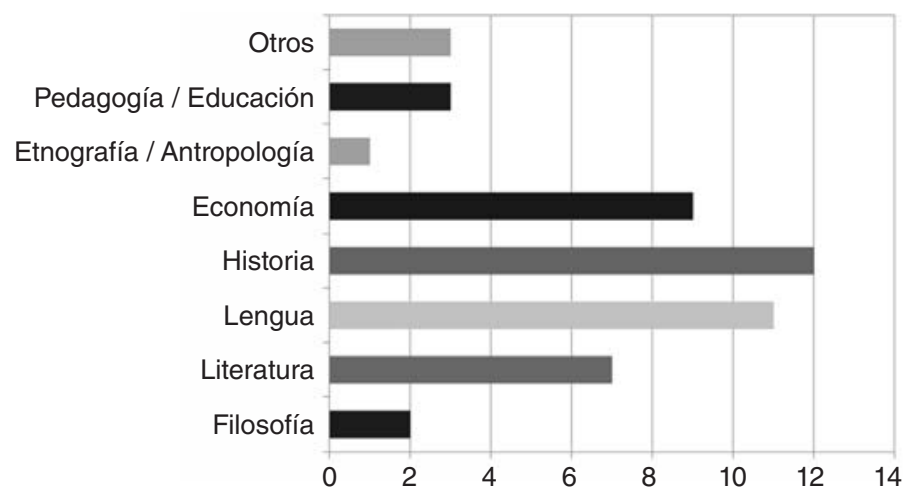

\begin{tabular}{|l|c|c|c|c|c|c|c|}
\hline & $\mathbf{1 9 6 8}$ & $\mathbf{1 9 6 9}$ & $\mathbf{1 9 7 0}$ & $\mathbf{1 9 7 1}$ & $\mathbf{1 9 7 2}$ & $\mathbf{1 9 7 3}$ & Total \\
\hline Filosofía & 0 & 0 & 0 & 0 & 1 & 1 & 2 \\
\hline Literatura & 0 & 1 & 3 & 2 & 1 & 0 & 7 \\
\hline Lengua & 3 & 0 & 2 & 3 & 3 & 0 & 11 \\
\hline Historia & 1 & 4 & 2 & 2 & 0 & 2 & 11 \\
\hline Economía & 4 & 2 & 0 & 2 & 1 & 0 & 9 \\
\hline Etnografía / Antropología & 0 & 0 & 0 & 0 & 1 & 0 & 1 \\
\hline Pedagogía / Educación & 0 & 1 & 1 & 1 & 0 & 0 & 3 \\
\hline Otros & 0 & 0 & 2 & 0 & 0 & 1 & 3 \\
\hline
\end{tabular}

Elaboración propia. 
FIGURAS 21 y 22

LA CREACIÓN FICCIONAL EN LA PRODUCCIÓN EDITORIAL

DE GALAXIA (1968-1973)

Tipología libros 1968-1973 (III). Producción literaria

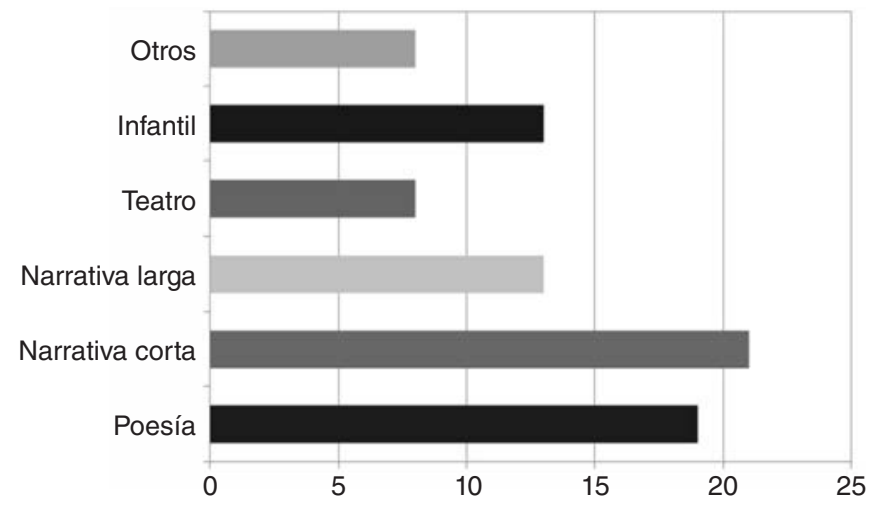

Tipología libros 1968-1973 (III). Producción literaria (totales)

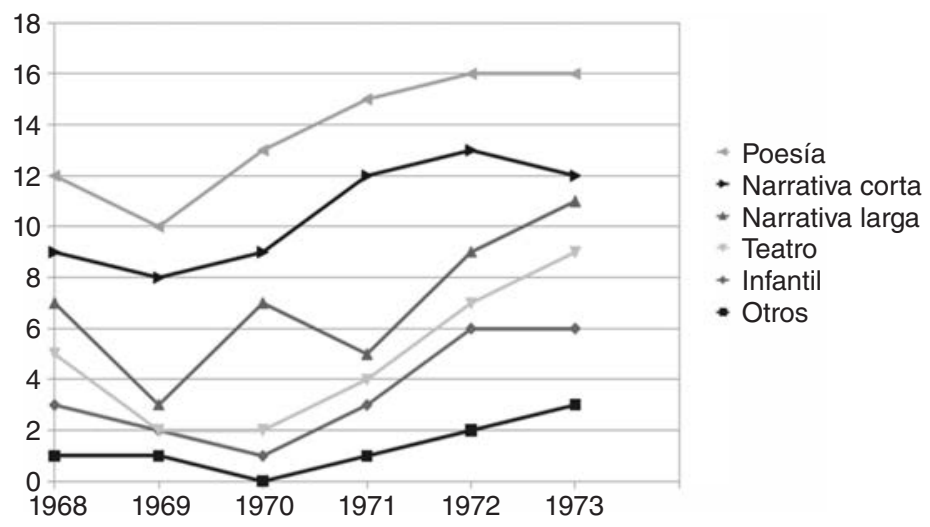

\begin{tabular}{|l|c|c|c|c|c|c|c|}
\hline & $\mathbf{1 9 6 8}$ & $\mathbf{1 9 6 9}$ & $\mathbf{1 9 7 0}$ & $\mathbf{1 9 7 1}$ & $\mathbf{1 9 7 2}$ & $\mathbf{1 9 7 3}$ & Total \\
\hline Poesía & 3 & 2 & 4 & 3 & 3 & 4 & 19 \\
\hline Narrativa corta & 2 & 5 & 2 & 7 & 4 & 1 & 21 \\
\hline Narrativa larga & 2 & 1 & 5 & 1 & 2 & 2 & 13 \\
\hline Teatro & 2 & 0 & 1 & 1 & 1 & 3 & 8 \\
\hline Infantil & 2 & 1 & 1 & 2 & 4 & 3 & 13 \\
\hline Otros & 1 & 1 & 0 & 1 & 2 & 3 & 8 \\
\hline
\end{tabular}

Elaboración propia. 
ción- de la aprobación de la Ley General de Educación de 1970 que anuncia la entrada del gallego en la enseñanza.

La confrontación entre las características de la producción ficcional publicada por Galaxia en el período 1968-1973 con la de etapas anteriores ofrece de nuevo notables diferencias [Figuras 21 y 22], principalmente en lo tocante al género poético que ve reducida su presencia proporcional desde más de la mitad de la producción hasta menos de un cuarto en esta etapa. En sentido contrario, la narrativa experimenta un notable avance en el total, continúa siendo muy mayoritaria la de extensión corta/media frente a la larga, y se consolida, además, como la modalidad genérica con mayor producción proporcional en el período tratado. Por otro lado, el teatro persiste en una situación deficitaria teniendo también en cuenta la escasa presencia de la producción (con solo tres obras: Un hotel de primeira sobre o río de Xohana Torres, 1968; Prisciliano: Traxidrama en dous aitos de Daniel Cortezón, 1970 y Catro pezas de Ricardo Carballo Calero, 1971) y el recurso como suplemento a la importación con las obras ya referidas y más secundariamente a la tradición, con un representante único pero con varias ediciones, Os vellos non deben de namorarse de Castelao. Finalmente y en lo relativo a los libros destinados al público infantil, debe ser anotada su consolidación definitiva con una mayoritaria producción de autoría gallega y correspondiente a las nuevas voces que empiezan a afianzarse en la época (como Arcadio López Casanova, Carlos Casares o Xohana Torres), junto con la presencia más ocasional de alguna traducción (ya no procedente del referente catalán) e, incluso, la fijación en texto de obras pertenecientes a la tradición oral, como ocurre en Don Gaifar e o tesouro (1970) o Cousas de Xan e Pedro (1971).

\section{CONCLUSIONES}

Los análisis expuestos permiten ofrecer algunas conclusiones globales sobre la actuación de Galaxia en el campo editorial gallego del período 1951-1973 $y$, en general y por la importancia de esta entidad dentro de él, sobre el propio sistema cultural en proceso de emergencia. El proyecto de la editorial surge en un momento en que comienza a haber una incipiente producción editorial en gallego con pequeñas casas editoras — como Bibliófilos Gallegos, la Colección de Poesía Benito Soto o la Editorial Monterrey_ que admiten o promueven la creación en gallego. El caso de Galaxia se diferencia de ellas por sus dimensiones y ambiciones, al tener una continuidad temporal no comparable a las anteriores, incorporar muy pronto labores de distribución e incluso de impresión a inicios de la década de 1970. A pesar de las cifras de obras editadas es relativamente pequeña, estas se mantienen constantes, lo que tiene un notable efecto en el sistema cultural del momento y contribuye a reforzar la identidad grupal de Galaxia. Existe en todo su programa una voluntad de rellenar los 
vacíos detectados, tanto en lo que concierne a una producción asociada a la alta cultura (sobre todo la filosofía que contribuía a legitimar el proyecto identitario gallego), como aquellos aspectos de la producción literaria percibidos como insuficientemente desarrollados (narrativa, teatro, literatura infantil, etc.), junto con la potenciación de aquellos materiales que se adapten a las nuevas circunstancias del campo y contribuyan a la llegada a un público mayor. Un ejemplo de esto último, más allá de la literatura infantil ya mencionada, son los contactos realizados con la discográfica barcelonesa Edigsa en 1966 para publicar discos en gallego en un momento en que comenzaba a surgir el movimiento de la Nova Canción gallega (Piñeiro y Losada, 2009: 45 y s.).

Los usos lingüísticos operados por Galaxia revelan la tendencia al uso del gallego, especialmente en la ficción y en menor medida en las publicaciones ensayísticas, dado que el régimen franquista era más tolerante en la primera modalidad mencionada. La progresiva extensión de uso de la lengua autóctona revela asimismo la propia apertura que se va produciendo en este aspecto desde el poder político. Futuros estudios sobre la actividad editorial de Galaxia deberían, aparte de agrandar el período de estudio, incorporar parámetros como número de ventas, precios, mecanismos de distribución, pago de derechos de autor, negociación de las traducciones o acción de la censura, datos hasta este momento difíciles de obtener pero que ofrecerían un panorama mucho más amplio y contribuirían a explicar mejor la actuación de Galaxia.

En lo que concierne al sistema cultural gallego en proceso de emergencia, las conclusiones se producen en tres niveles: por una parte, la presencia mayoritaria en la producción editorial de Galaxia de obras ficcionales (frente al resto de temáticas), revelando un sistema altamente literaturizado; en segundo lugar, se constata la extensión progresiva del campo, con un aumento de la producción y de intervinientes, lo que es paralelo a la estrategia de legitimación identitaria puesta en marcha por el grupo Galaxia a diferentes niveles; finalmente, se detecta una evolución en la presencia e interpretación de los géneros literarios, pasando de una primera etapa con un peso fuerte de la poesía, en consonancia con las prácticas habituales identificadas en procesos de emergencia (Even-Zohar, 1994), para surgir después un protagonismo de la narrativa, cambiando las funcionalidades atribuidas y simbolizando con ello un proceso de consolidación sistémica.

Por último, los parámetros elegidos para realizar el análisis de Galaxia pueden ser fácilmente exportables a contextos análogos permitiendo realizar un estudio profundo de la producción editorial que contribuya a explicar la propia posición y actuación de las casas editoriales, pero también la evolución del campo. En este sentido, situaciones semejantes a las identificadas en el espacio social gallego durante el período propuesto en que produce una coexistencia no pacífica de sistemas culturales, español y gallego, con diferente entidad y distribución, son comparables a las experimentadas en Cataluña o Quebec, y por eso mismo susceptibles de ser abordados desde estos mismos prismas de análisis. 


\section{BIBLIOGRAFÍA CITADA}

Beiras Torrado, Xosé Manuel (2005). «Revista de economía de Galicia», en Benxamín Casal Vila (dir.), Gran Enciclopedia Galega Silverio Canada [edición en CD-R]. Lugo: El Progreso, Diario de Pontevedra.

Beramendi, Justo G. y Xose Manoel Núñez Seixas (1996). O nacionalismo galego. 2. a ed. corregida y aumentada. Vigo: A Nosa Terra.

Bourdieu, Pierre (1992). Les Reglès de l'art. Genèse et structure du champ litteraire. Paris: Seuil.

Cordeiro Rua, Goncalo y Roberto Samartim (2008). «O panorama editorial galego no tardofranquismo e na transiçom», en Paz Romero Portilla y Manuel-Reyes García Hurtado (coord.), El libro en perspectiva. Una aproximación interdisciplinaria. A Coruña: Universidade da Coruña, pp. 161-193.

Dasilva, Xosé Manuel (2007). «As traduccións ibéricas de Os vellos non deben de namorarse», en Helena Gonzalez y M. ${ }^{a}$ Xesus Lama (ed.). Actas do VII Congreso Internacional de Estudos Galegos. Mulleres en Galicia. Galicia e os outros pobos da Península. Barcelona 28 o 3 de maio de 2003. Sada, Barcelona: Ediciós do Castro / Universitat de Barcelona, pp. 949-969.

Editorial Galaxia (1970). Catalogo xeneral de publicacios 1971. Vigo: Galaxia.

Editorial Galaxia (1973). Catalogo xeneral de publicacios 1974. Vigo: Galaxia.

Editorial Galaxia (1974). Almanaque Galaxia 1950-1975. Vigo: Galaxia.

Even-Zohar, Itamar (1990). «Polysystem Theory», Poetics Today. 11, pp. 27-96.

Even-Zohar, Itamar (1994). «La función de la literatura en la creación de las naciones de Europa», en Darío Villanueva (ed.). Avances en Teoría de la literatura: Estética de la Recepción, Pragmática, Teoría Empírica y Teoría de los Polisistemas. Santiago de Compostela: Universidade de Santiago de Compostela, pp. 357-377.

Even-Zohar, Itamar (2010). Papers in Culture Research. Tel Aviv: The Culture Research Laboratory. Disponible en: <http://www.tau.ac.il/ itamarez/works/books/EZCR-2005_2010.pdf $>$ [ref. de 23/02/2015].

Fernández del Riego, Francisco (1996). A xeración Galaxia. Vigo: Galaxia.

Fernández del Riego, Francisco (2000). Un epistolario de Ramón Piñeiro. Vigo: Galaxia.

Figueiras, Carlos G. (2009). "A literatura infanto-juvenil galega no tardo-franquismo», en Carmen Villarino Pardo, Elias J. Torres Feijó y José Luís Rodríguez (ed.). Da Galiza a Timor. A lusofonia em foco. [Actas do] VIII Congresso da Associação Internacional de Lusitanistas. Santiago de Compostela: Universidade de Santiago de Compostela, vol. III, pp. 1973-1982.

Lambert, José (1980). «Production, tradition et importation: une clef pour la description de la littérature et de la littérature en traduction», Canadian Review of Comparative Literature / Revue Canadienne de Litterature Comparée. 7 (2), pp. 246-252.

Lambert, José (1983). «L'eternelle question des frontieres: litteratures nationales et systemes littéraires», en Christian Angelet, Ludo Melis y Frans Jozef Mertens (ed.). Langue, Dialecte, Litterature. Études romanes à la memoire de Hugo Plomteux. Lovaina: Leuven University Press, pp. 355-370.

Martínez Martín, Jesús A. (2015). «Editoriales y libros en catalán, gallego y euskera», en Jesús A. Martínez Martín (dir.), Historia de la edición en España (1939-1975). Madrid: Marcial Pons, pp. 835-858.

Núñez Seixas, Xosé Manoel (1994). «Galeguismo e cultura durante o primeiro Franquismo (1939-1960). Unha interpretacion (e II)», A Trabe de Ouro. 4/ 20, pp. 85-103. 
Piñeiro, Ramón y Basilio Losada (2009). Do sentimento á conciencia de Galicia. Correspondencia (1961-1984). María Xesús Lama y Helena Gonzalez (ed.). Vigo: Galaxia.

Samartim, Roberto (2005). «Ideia de língua e vento português na Galiza do tardofranquismo: O caso de Galaxia», Agália. 83/84, pp. 9-53. Disponible en: <http://www.agal-gz.org/ faq/lib/exe/fetch.php?media=agalia:n83-84_ideia_de_lingua_e_vento_portugues_na_galiza.pdf> [ref. de 20/07/2015].

Samartim, Roberto (2010). O processo de construçom do sistema literário galego entre o franquismo e a transiçom (1974-1978). Margens, relaçons, estrutura e estratégias de planificaçom cultural [Tesis doctoral]. Santiago de Compostela: Universidade de Santiago de Compostela.

Torres Feijó, Elias J. (2004): «Contributos sobre o objecto de estudo e metodologia sistémica. Sistemas literários e literaturas nacionais», en Anxo Abuín y Anxo Tarrío (coord.): Bases Metodolóxicas para unha Historia Comparada das Literaturas da Península Ibérica. Santiago de Compostela: Universidade de Santiago de Compostela, pp. 423-444.

Torres Regueiro, Xesús (2010). «A estrea na Galiza de Os vellos non deben de namorarse», Anuario Brigantino. 33, pp. 423-440.

Fecha de recepción: 23 de febrero de 2015.

Fecha de aceptación: 26 de mayo de 2015. 